\title{
Using Digital Micro-network Interactive System to Improve the Quality of Experimental Teaching of Drosophila Salivary Gland Chromosomes
}

\author{
Huang Tiran ${ }^{1,2}$, Guo Bei ${ }^{1}$, Lu Heshu ${ }^{1}$, Huang Huang ${ }^{1}$, Wang Cong ${ }^{1}$, Wang Shuying ${ }^{1}$ \\ ${ }^{1}$ College of Biological Sciences and Engineering, Beijing University of Agriculture, Beijing, China \\ ${ }^{2}$ Key Laboratory of Urban Agriculture (North China), Ministry of Agriculture P. R. China, Beijing, China
}

Email address:

huangtr151@bua.edu.cn (Huang Tiran)

${ }^{*}$ Corresponding author

To cite this article:

Huang Tiran, Guo Bei, Lu Heshu, Huang Huang, Wang Cong, Wang Shuying. Using Digital Micro-network Interactive System to Improve the Quality of Experimental Teaching of Drosophila Salivary Gland Chromosomes. Science Journal of Education. Vol. 9, No. 3, 2021 , pp. 73-76. doi: 10.11648/j.sjedu.20210903.11

Received: April 17, 2021; Accepted: May 10, 2021; Published: May 24, 2021

\begin{abstract}
Background: With the microscopic interactive system widely used in experiment teaching of Genetics, Botany, Cell biology, Medical morphology, etc., which are focused on the microscopic observation, Traditional experimental teaching mode is changed, experimental teaching efficiency and experimental teaching effect are improving. Objective: In order to improve the efficiency and effect of genetics experiment teachingthis, we introduce the digital micro-network interactive system into the genetics experiment teaching. Method: We implemented the observation and preparation techniques of Drosophila salivary gland chromosomes from teaching organization, implementation of the experiment, using the digital micro-network interactive system. And the application effect of microinteractive system in chromosome section of Drosophila salivary gland is analyzed. Result: The results showed that the microscopic interaction system could improve the efficiency and effectiveness of the interaction between teachers and students, stimulate the students' experimental interest. Especially, the success rate of salivary gland chromosome is increased by more than $20 \%$. Conclusion: The application of micro-interactive system has indeed revolutionized the traditional experimental teaching mode to a certain extent. It closely relies on modern information technology and forms a new experimental teaching mode for the cultivation of genetically innovative talents, which characterize by zero barriers in teacher-student interaction, zero distance in resource sharing and zero gap in real-time monitoring.
\end{abstract}

Keywords: Genetics Experiment, Microscopic Interaction, Chromosome Preparation, Drosophila Salivary Gland

\section{Introduction}

Genetics is a discipline studying the laws of biological heredity and variation, and is an important professional basic course in the field of life sciences. Experimental teaching plays an important role in cultivating and improving students' comprehensive quality, practical ability and innovative thinking ability [1]. With the advent of the information age, the application of computer network technology, multimedia technology, image acquisition and analysis technology, and micro-interaction technology in experimental teaching has become more and more extensive and in-depth [2].

Microscopic observation is an indispensable and effective method for morphological observation in multiple courses such as genetics [2], botany [3], cell biology [4], and medical morphology [5]. However, due to the lack of camera and interactive systems in traditional microscopes, the typical images observed by students cannot be shared by the whole class, which has caused great obstacles to the interaction of microscopic images between teachers and students [6]. Teachers can only frequently shuttle between students for one-on-one guidance. A lot of common problems encountered in the experiments frequently require repeated guidance many times, which invisibly increases the teacher's labor intensity. When many students ask questions at the same time, a lot of valuable classroom learning time was 
wasted while waiting for the teacher's guidance, because teachers should guide the students one by one [7]. Reforming the model of traditional experimental teaching, introducing a microscopic interactive teaching system, increasing the intuition, pertinence, convenience and effectiveness of teacher-student interaction, and improving the efficiency and effectiveness of experimental teaching, has become an urgent problem to be solved in life sciences morphology teaching. Micro-network interactive system is a new means of modern laboratory teaching. It is a networked and open interactive experimental teaching system, which uses digital technology and network transmission to organically connect the students' microscopes, teachers' microscopes and computers in the laboratory. It has powerful functions and is convenient to operate. The micro-network interactive system integrates the advantages of multimedia, computer and network technology, and has the characteristics of sharing, timeliness and interactivity point. In this paper, the observation of drosophila salivary gland chromosome are used as an example to introduce the application effect of the microscopic interactive system in genetics experimental teaching, in order to provide a useful reference for the experimental teaching of related subjects.

\section{Organization and Implementation}

\subsection{Teaching Organization of Drosophila Salivary Gland Chromosomes}

First, review and introduce the concepts and the structural characteristics of salivary gland, then use the multimedia projection system to show the salivary gland chromosomes to the students, and send the salivary chromosome image to every students through the microscopic interactive system. Based an overall understanding of chromosomes, How can we quickly obtain the third instar larvae of Drosophila is necessary? Finally, the purpose and requirements of this experiment are emphasized, and students are reminded to submit 2-3 pictures of the salivary gland chromosomes through the micro-interactive system after the experiment.

\subsection{Implementation of Drosophila Salivary Gland Chromosomes}

Larvae culture: Put the drosophila in a nutrient-rich medium and raise them at $16-18^{\circ} \mathrm{C}$. When the larvae climb on the bottle wall and prepare for pupation, they are the third instar larvae. Select the larvae that are growing and hypertrophic, and dissect them.

Take the salivary gland: select the growing and hypertrophic larva and place it on a glass slide, drop 1-2 drops of $0.7 \%$ sodium chloride aqueous solution, find the head with mouthparts under the dissecting microscope, and fix the worms by piercing the back of the mouthparts with one hand with a dissecting needle, Clamp the lower half of the larva with tweezers in one hand, and then use the dissecting needle to pull the head forward from the front of the worm body, and the salivary gland are pulled out with the head. The salivary gland is cystic and translucent, with fat bands attached to them, one on each side of the esophagus, and the cells are clearly separated and arranged neatly. Use tweezers to remove the rest of the worm body, carefully pick out the fat body adhering to the salivary gland, carefully wash it, and absorb the residue and excess water with absorbent paper.

Staining and slide preparation: Put a drop of $1 \mathrm{~mol} / \mathrm{L} \mathrm{HCl}$ on the gland and soak for $2 \sim 3$ minutes. Slowly pour off the $\mathrm{HCl}$, rinse carefully with water, and soak dry while dripping. After the water is soaked up, add a drop of acetic magenta for staining for a few 30 minutes, add a cover slip, cover the cover slip with absorbent paper, and then use your thumb to press the tablet properly.

Microscopic examination: Place the compressed film under the dissecting microscope to observe the chromosome staining center, chromosome arms, horizontal stripes, etc. of the salivary gland..

\section{Application Effect Analysis}

\subsection{Real-Time Video Monitoring Powerfully, Improving the Efficiency of Guidance Greatly}

In the traditional microscopic observation experiment teaching, every students hand a microscope to observe, each student's contents under the microscope cannot be seen at any time by the teacher. Therefore, whether the student can find the object under the microscope accurately, whether he understands the object to be observed, or even teachers cannot fully know whether they are observing [8]. Using the microscopic interactive system, teachers can monitor the student's microscope image in real time, monitor a student's screen in full screen at any time, and can obtain the student's mouse and keyboard control through the remote tutoring function, and remotely operate the student's computer. Step off the stage, you can remotely tutor any student, and realize online teaching. If students violate disciplines, teachers can choose to use "black screen and silence" to warn students of lack of concentration such as surfing the internet and playing games during class time [9], and conducive to maintaining classroom order. Teachers also can check the student's production process at any time, discover and solve and correct irregular operations in the student experiment in time, which is conducive to real-time monitoring of teaching quality. More importantly, the mirror image can be projected to the big screen through the multimedia system to explain to the whole class, or to select a common problem or typical image that one student appears in the film production process for teaching, and observe within the whole class discussion, experimental works can be submitted in the classroom, and the efficiency of teachers' teaching and guidance has been greatly improved [10]. The use of micro-network interactive system not only lightens the workload of teachers, but also sets up a good atmosphere of interaction between teachers and students, which activates the classroom atmosphere and stimulates students' interest in learning [11]. 


\subsection{Teacher-Student Interaction Fast and Efficiently, Improving the Quality Of Teaching Greatly}

The salivary gland chromosomes of drosophila are often adhered to the surrounding fatty tissues, nerves and esophageal tubes, it is difficult to distinguish them. For the beginners, it is easy to confuse them with other surrounding tissues. Under the traditional teaching mode of microscopy experiments, common problems need to be repeated many times. This not only increases the labor intensity of teachers, but also causes a lot of class time waste [7-8]. With the application of the microscopic interactive system, images under the microscope observed by the students can be transmitted to the teacher's computer as soon as possible. Then the teacher can provide targeted guidance in real time. If the salivary gland have been stripped out under the microscope, the target object can be pointed out with the help of mouse at the first time in order to reduce the frequency and number of times of the teacher's patrols among students, the teaching efficiency is greatly improved [9-10]. For the transient structure under the mirror, it can take photos and store them in real time to supplement teaching resources. According to incomplete statistics, before the microscopic interactive system, this salivary gland chromosomes experiment was the longest time-consuming of all genetic experiments, because most of the time was spent on the stripping and picking of salivary gland chromosomes. Due to tight time, the quality and effect of the filming are not ideal. About $30 \%$ of the students have unclear salivary chromosomes or lost chromosomes during the filming process. After using the microscopic interactive system, the experiment time was reduced by about $25 \%$, and the proportion of unclear chromosomes or material loss fall less than $10 \%$. In a word, After introducing the micro-network interactive system, it can effectively avoid the repetition of experimental problems and share excellent experimental results in a timely manner, thus greatly improving the teaching efficiency in the limited class hours [12].

\subsection{Utilizing Broadcast Teaching, Innovating Teaching Mode Table}

Through the broadcast teaching function, the sound and image can be transmitted to each student's computer screen in real time and smoothly, or the sound and image can be displayed on a large screen projection. In the observation and preparation of drosophila salivary gland chromosome experiment, beginners generally lack an intuitive understanding of drosophila salivary gland. Through this teaching mode, the teacher can transmit the drosophila salivary gland stripping video and drosophila salivary gland stripping anatomy to the student computer, students could watch and practice. This not only improves the pertinence of teachers' teaching, but also improves the intuition of teachers' teaching content, and can be laying a solid foundation for the cultivation of innovative talents in genetics. Most importantly, the broadcast teaching allows each student to sit in front of their own screen and observe every step of the teacher's operation, which further improves the teaching method and widens and enriches the teaching content and resources on display [12-13]. In addition, through micro-network interactive system, teachers can more comprehensively observe the experimental operation process of students and the performance of answering questions, and better understand the differences in the usual performance of each student so as to achieve fairness and justice, which has laid a solid foundation for improving the examination system of experimental course [14].

\subsection{Stimulate Students' Interest Of Experiments Using Image Acquisition System}

Using of the microscopic interactive system can transmit the under-mirror image to the computer display screen through the CCD camera, and then the eyes can be freed from the eyepieces. This improves the student's highly concentrated state greatly when looking at the microscope during taking salivary gland, reduces eye fatigue effectively, and helps to find and strip the salivary gland of fruit flies quickly. With the enough time for the subsequent production of salivary gland chromosomes, the success rate of production can be improved, and then students' interest in experiments can be stimulated. Especially, it is helpful for students to discuss and communicate with each other, strengthen students' understanding of the teaching content in class, and cultivate students' innovative thinking [14-16].

\section{Conclusion}

By using the digital micro-network interactive system, teachers can collect the images of the typical section of the drosophila salivary gland chromosome in advance according to the teaching content of each lessons, and then make the lantern slide. It can be used directly in the teaching of theory and experiment in order to save time and effort. Of course, these teaching contents can also be demonstrated by the teachers personally in the experimental class, and the students can intuitively and clearly observe the operating contents through the screen of the student-side computer, so as to understand the teaching content more accurately [17]. Experimental teaching is to deepen the understanding and application of theoretical knowledge in the process of practice through students' hands-on operation, thinking and discussion. Its importance cannot be ignored [18]. With the application of the microscopic interactive system, the traditional experimental teaching mode has revolutionized to a certain extent. Relying on modern information technology, a new model with zero barriers to teacher-student interaction, zero distance for resource sharing, and zero gaps in real-time monitoring has been formed. The experimental teaching mode has significantly improved the efficiency of teacher guidance and the quality of teaching, and stimulated students' interest in experiments. $。$ With the development of modern information technology, the function of the microscopic interactive system will become more and more perfect, and it will play an increasingly important role in experimental teaching. 


\section{Acknowledgements}

This study was supported by grants from scientific research project of Beijing Municipal Education commission (KM201910020016).

\section{References}

[1] T. R. Huang, B. Guo, Y. F. Liu, H. S. Lu, Y. Z. Sun, and X. H. Shen. Reforming Genetics Experiment Teaching for Improving the Quality of Personnel Training. Experiment Science and Technology, 2017, 15 (3): 91-94.

[2] Z. H. Tang, Y. F. Mao, X. D. Zheng, and L. F. Kong. Application effect of microscopic interactive multimedia system in genetics experimental teaching. Experiment Technology and Management, 2011, 28 (11): 85-87.

[3] J. J. Bi, B. X. Ye, A. Y. Chang, R. Zhang, X. W. Qiu, J. L. Li, and J. H. Wang. The application of digital net work of microscope laboratory to teaching of biological experiment. Research and Exploration in Laboratory, 2007, 26 (5): 51-53.

[4] X. B. Wang, and P. Wang. Appication of the microscopic digital aystem in the experiment teaching of cell biology. Laboratory Science, 2011, 14 (6): 65-66.

[5] J. J. Ni., Z. G. Ying., and W. Yao. Application of Digital Microscopic Interactive Laboratory in Medical Morphology Experiment Teaching. China higher Medical Education, 2005, 3: 80-81.

[6] L. L. Yue, L. H. Zheng, P. Chen, H. T. Yu, D. Liu, Y. X. Lu. Practice and exploration of the integration of information technology and medical genetics. Journal of Medical Reaearch, 2010, 39 (6): 127-128.

[7] H. X. Su, H. W. Shang, L. X. Zhang, X. Lu, P. Jing, and X. L. Tong. Application and prospectof digital-internet microscopic mutual motivation system for the experimental morphology teaching. Basic and Clinical Medicine, 2008, 28 (6): 651-653.

[8] H. L. Zhou, D. Z. Zhang, X. X. Hao, and W. Z. Liu. The composition and application of the interrative digital microscope laboratory in the experimental teaching of morphology. Acta Academ Iae Medicinae Neimongol, 2009, 31 (1): 122-124.

[9] J. H. Cheng, Z. Rong, Y. H. Wang, and L. Y. He. Application and retrospective analysis of digital network micro interaction system in morphology experiment teachin. Chinese Medical Equipment Joural, 2014, 10: 130-132.

[10] F. J. Shao, Y. W. Leng, H. W. Zhu, and L. Xiao. Application of microscopicmutual laboratoryin the teaching of microbial experiment. Laboratory Science, 2012, 15 (5): 123-125.

[11] M. J. Wang, H. Y. Zhu, and F. Chen. Practice of microscope digital mutual system in cell biological experimental course. China Continuing Medical Education, 13 (4): 1-4.

[12] R. M. Li, Q. Du, G. T. Peng, Z. D. Xu, and J. M. Chen. Application of digital microscope interactive system in experimental teaching of pharmaceutical botany. Research and Exploration in Laboratory, 39 (9): 232-234.

[13] J. H. Chen. Y. Wang, and S. Y. Wang. Teaching method of microbiology experiment based on the motic digital interactive system. Laboratory Science, 2019, 22 (1): 89-92.

[14] Q. H. Yu, L. X. Yuan. The Application of Virtual Simulation and Digital Network Micro-interactive System in Histomorphology Experimental Teaching. Experiment Science and Technology, 2018, 16 (3): 54-56.

[15] H. Y. Chen, X. X. Chen, and Y. Huang. Application of Digital Microscopic Interactive System in Genetics Experiment Teaching. Biology Teaching in University, 2018, 8 (2): 52-54.

[16] S. L. Bo, T. F. Ma, Z. Li, M. Zhou, J. L. Zhao, and H. Yan. Application of microdigital interactive system in histology experiment teaching. Anatomy Research, 2020, 42 (6): $560-561$.

[17] J. Cao, J. H. Tao, B. Q. Hu, L. Q. Chen, W. P. Zhou, and C. F. Xie. Study on Application of Digital Microscope Mutual System in the Experimental Teaching of " Magmatic Petrology". Jounal of East China University of Technology (Social Science), 2020, 39 (5): 492-495.

[18] R. Guo, T. Zhang, Y. Yuan, and X. H. Wang. Feedback survey analysis of applycation of motic digital microscopy interactive lab. China Higher Medical Education, 2019, 12: 34-35. 